\title{
CORTICOTERAPIA PRÉ-NATAL NAS SÍNDROMES HIPERTENSIVAS DA GESTAÇÃO E SEUS EFEITOS NA PRESSÃO ARTERIAL MATERNA
}

\author{
*N. Sass, R.R. Cançado, M.L. Oliveira, M.R. Torlonı
}

Trabalho realizado no Hospital e Maternidade Escola Dr. Mário de Moraes Altenfelder Silva, São Paulo, SP

RESUMO - OBJetivos. Avaliar o comportamento da pressão arterial em gestantes portadoras de síndromes hipertensivas na vigência de ciclo de corticóide (esquema de LIGGINS) utilizado para a aceleração da maturidade pulmonar fetal.

Métodos. A partir de estudo retrospectivo, foram avaliadas 27 gestantes portadoras de hipertensão arterial, com idade gestacional entre 24 e 34 semanas, submetidas a corticoterapia antenatal. Para tanto, foi realizada análise estatística das médias das pressões arteriais sistólicas e diastólicas separadamente, dos dias anterior, primeiro e segundo dias que compõem o ciclo de corticoterapia e do dia posterior a este ciclo. Obtidas as variâncias, foi aplicado o teste F Statistic, analisado através do valor de $p$, significante, se menor que 0,05 .

Resultados. Não foi observada variação significativa dos níveis de pressão arterial, seja sistólica como diastólica, não se identificando dificuldades no controle clínico das pacientes, não tendo sido observada necessidade de elevação das doses de drogas hipotensores utilizadas.

ConcLusÃo. Nossos resultados observaram segurança na utilização de ciclos de corticóides em pacientes portadoras de hipertensão arterial em relação à possíveis agravos dos níveis pressóricos.

Unitermos: Hipertensão arterial na gestação. Prematuridade. Corticoterapia pré-natal.

\section{INTRODUÇÃo}

No início da década de 70, a administração de corticóides em experimentação animal refletiu em uma ação efetiva na aceleração da capacidade funcional de pulmões de recém-nascidos prematuros', reduzindo de maneira significativa a incidência da síndrome do desconforto respiratório, com redução evidente nas taxas de mortalidade neo-natal.

Seguindo-se a divulgação destes resultados, vários trabalhos foram desenvolvidos onde se procurava estabelecer de maneira definitiva sua ação benéfica e possíveis reações adversas, possibilitando estender seu uso na rotina diária de assistência ao parto prematuro. Baseada nas melhores evidên-

*Correspondência: Nelson Sass

Rua Umberto Boccioni, 210 - Cep: 02441-150 Fax: 6231-9969

São Paulo - SP - E-mail: nelsonsa.alp@ zaz.com.br cias, acumuladas na experiência anterior de vários trabalhos ${ }^{2}$, sua utilização passa a ser recomendada pelo Instituto de Saúde dos Estados Unidos em consenso estabelecido por equipe multidisciplinar como norma na assistência à prematuridade 3 .

Ressalte-se ainda as conseqüências do ponto de vista econômico que esta terapêutica pôde acarretar. Os custos relacionados ao atendimento ao RN prematuro em unidades de terapia intensiva é extremamente elevado, somando-se ainda os gastos decorrentes das complicações tardias da prematuridade. Estima-se que nos EUA, haveria uma economia de 157 milhões de dólares a cada ano, apenas considerando a redução de tempo de permanência hospitalar em unidades de terapia intensiva.

Levando-se em conta que a prematuridade representa para o Brasil problema de extrema importância, particularmente relacionada à hipertensão arterial materna, a utilização de rotina da corticoterapia prénatal pode contribuir de forma efetiva para a redução de custos em um sistema de saúde permanentemente deficitário.

Ainda que existam vantagens clínicas e econômicas justificando sua utilização, verifica-se, muitas vezes, resistência ao seu uso como rotina, particularmente em situações em que se verifique a presença de hipertensão arterial materna, diabete melito ou rotura prematura das membranas ovulares.

Em relação aos estados hipertensivos, um dos fatores a limitar sua utilização foi baseado nos resultados divulgados nos trabalhos iniciais', onde se registrava maior prevalência de óbitos fetais quando comparadas as taxas verificadas no grupo controle. Outro fator relaciona-se a uma certa "convicção", que a hipertensão arterial auxiliaria per se, a maturação pulmonar. Tal assertiva não encontra base científica de sustentação, uma vez que recém-nascidos prematuros 
de mães hipertensas enfrentam sérios problemas de adaptação à vida neo-natal. Observa-se ainda, como fator limitante, o temor relacionado a possibilidade de agravamento dos níveis pressóricos maternos quando da ministração da droga.

Em vista de ser este fator motivo muito freqüente em nosso meio para que se adie ou mesmo não se utilize de ciclos de corticóides, tivemos como objetivo neste trabalho observar a influência destes (esquema de LIGGINS) na pressão arterial de gestantes portadoras de hipertensão arterial.

\section{Métodos}

Trata-se de estudo retrospectivo constituído de 27 gestantes portadoras de síndromes hipertensivas, com idade gestacional entre 24 e 34 semanas, que utilizaram corticoterapia para maturação pulmonar fetal, internadas na Casa da Gestante de Alto Risco do Hospital e Maternidade Escola Mário de Moraes Altenfelder Silva (Vila Nova Cachoeirinha), no período de janeiro de 1998 a agosto de 1998.

O ciclo de corticóide consistiu na ministração de 12,0 mg de betametasona intramuscular em dois dias consecutivos, segundo esquema de LIGGINSI.

A internação destas pacientes foi determinada pela necessidade de acompanhamento de situações clínicas maternas de risco, como hipertensão arterial grave e/ou sinais de insuficiência placentária, necessitando de observação prospectiva das condições da vitalidade fetal. Tal permanência não significava necessariamente a resolução do parto, tendo este ocorrido em tempo variável em relação a ministração dos ciclos, sendo possível em alguns casos a alta da paciente em vista da estabilização do quadro clínico.

Foram analisados os prontuários e os respectivos registros de pressão arterial em cada paciente e a eventual necessidade de elevação das doses de drogas hipotensoras utilizadas. As tomadas de pressão arterial foram obtidas nos horários de $2 \mathrm{~h}$, 8h, I 4h e 20h, diariamente, utilizando-se de esfigmomanômetro de coluna, no braço esquerdo, com as pacientes sentadas no leito, durante os controles de rotina, tendo como parâmetro de pressão diastólica o $5^{\circ}$ ruído de Korotkoff.

Foram calculadas médias aritméticas simples das pressões arteriais sistólicas e diastólicas, obtidas no dia anterior e posterior ao ciclo e em cada um dos dois dias deste. Com estes dados, foi aferida a variância, que traduziu a resposta do grupo em estudo à exposição de determinado fator, $\frac{\sum(X \mid-X m)^{2}}{N-1}$

$\mathrm{N}-\mathrm{I}$ onde $\sum$ significa somatória das pressões arteriais $(X I)$ sistólicas e diastólicas separadamente, uma a uma, subtraídas da média das pressões arteriais (Xm) de cada um dos dias após elevá-las ao quadrado. $\mathrm{N}$ corresponde ao número de pacientes multiplicado pelo número de aferições diárias de pressão arterial.

Após obtidas as variâncias, foi aplicado o teste F Statistic, com uso do programa Epi Info 6.04®. O F Statistic foi analisado com p significante se menor que 0,05.

\section{Resultados}

Nas tabelas | e 2, observa-se a variância de pressão sistólica e diastólica, respectivamente. Não houve diferença significante, quando se comparam as médias de pressão no dia anterior ao ciclo de corticóide, nos dois dias que o compõem e no dia posterior a este ciclo, tanto para as pressões sistólicas quanto para as diastólicas. $O$ esquema utilizado não apresentou influências em termos de agravamento dos níveis pressóricos maternos no período estudado, não se observando a necessidade de elevação das doses de hipotensores.

\section{Discussão}

A utilização de corticóides visando a aceleração da maturidade pulmonar em fetos prematuros, constitui ação evidentemente eficaz em termos de redução da morbidade e mortalidade neonatal. Em 1994, o Instituto Nacional de Saúde dos EUA estabeleceu um consenso relativo ao uso de corticosteróides para a maturação pulmonarvisando melhor desempenho perinatal, que devem ser ressaltados e ampliados à prática diária ${ }^{3}$.

A terapia com corticóides no período pré-natal reduzem a mortalidade, a síndrome de desconforto respiratório transitório e hemorragia cerebral em recémnascidos pré-termo. Estes benefícios ocorrem quando administrados no intervalo entre 24 e 34 semanas, independente da idade ou raça materna ou do sexo fetal. 0 efeito benéfico do corticóide é potencializado quando da utilização concomitante de surfactante, sendo que os possíveis riscos maternos superam em muito as vantagens que o método proporciona emtermos de possibilidade de sobrevida neo-natal.

Ainda que os relatos iniciais' alertassem para possíveis riscos em sua utilização em pacientes portadoras de síndromes hipertensivas, as evidências que se seguiram indicavam outra direção, associando a maior mortalidade neste grupo de pacientes às condições adversas da própria patologial; 4:5.

A publicação dos resultados obtidos pelo Grupo Colaborativo de Terapia Esteróide Antenatal ${ }^{6}$ além de registrar a redução na incidência de desconforto respiratório no grupo tratado, consideraram que não houve influência significativa em patologias intercorrentes na gestação, tais como a hipertensão arterial.

Ainda assim, nos dias atuais verificam-se graus variáveis de resistência à utilização sistemática dos esquemas preconizados, particularmente em relação à possibilidade do 
agravamento da pressão arterial materna, abdicando-se de método que pode ser diferencial na qualidade da evolução perinatal.

Teoricamente, os glicocorticóides podem afetar o curso da hipertensão em gestantes devido às suas atividades mineralocorticóides, que acarretariam elevação dos níveis pressóricos. Porém, tanto a betametasona como a dexametasona representam uma classe de fluorocorticóides com ação mineralocorticóide mínima ou ausente ${ }^{7}$, praticamente sem nenhum impacto em termos de expansão volumétrica, ambos com atividade biológica similar, atravessando a barreira placentária e atingindo níveis adequados no compartimento fetal para sua ação farmacológica. As doses preconizadas são sintetizadas no quadro l.

No que diz respeito a polêmica relacionada ao agravamento da pressão arterial ou de dificuldade no controle dos níveis pressóricos maternos diante da administração da droga, verificam-se ensaios onde não se registraram tal fenômeno $0^{4,8,9}$.

Em nosso meio, estudo relacionado ao uso de corticóides para a aceleração da maturidade pulmonar fetal em pacientes portadoras com pré-eclampsia grave, também não registrou alterações significativas na pressão arterial materna, tanto na pressão sistólica como na diastólica, que pudessem acarretar algum prejuízo materno relacionado a sua administração. Além disso, a autora registra redução de 50,0\% na incidência de doença de membrana hialina entre os recém-nascidos que receberam a terapia pré-nata|l0.

Nossos resultados vão ao encontro aos relatos da literatura, sendo que o comportamento da pressão arterial entre as pacientes não mostrou alterações que pudessem ser associadas à droga. Em nenhum caso houve a necessidade de modificações nas doses de hipotensores utilizados. O momento do parto em relação a administração dos ciclos dependeu das condições da circulação úteroplacentária, sendo que 44,0\% dos recém-

Tabela I - Análise da variância para pressão arterial sistólica entre gestantes hipertensas submetidas à corticoterapia pré-natal (H. Vila Nova Cachoeirinha, Janeiro a Agosto, 1998).

\begin{tabular}{cccc}
\hline dia & média & variância & número de medidas x pacientes \\
anterior & 143,05 & 314,92 & 108 \\
$1^{\circ}$ dia & 145,92 & 361,92 & 108 \\
$2^{\circ}$ dia & 142,87 & 389,11 & 108 \\
posterior & 143,70 & 489,15 & 108 \\
\hline
\end{tabular}

Valor de p: 0,650672 ( significância se $p<$ que 0,05)

Tabela 2 - Análise da variância para pressão arterial diastólica entre gestantes hipertensas submetidas à corticoterapia pré-natal (H. Vila Nova Cachoeirinha, Janeiro a Agosto, 1998).

\begin{tabular}{cccc}
\hline dia & média & variância & número de medidas x pacientes \\
anterior & 94,12 & 153,19 & 108 \\
$1^{\circ}$ dia & 92,77 & 163,44 & 108 \\
$2^{\circ}$ dia & 90,50 & 170,90 & 108 \\
posterior & 90,50 & 267,63 & 108
\end{tabular}

Valor de p : 0,141379 (significância se $p<$ que 0,05)

\begin{tabular}{lccc}
\hline \multicolumn{4}{c}{$\begin{array}{l}\text { Quadro I - Doses preconizadas para tratamento pré-natal } \\
\text { com corticóides entre } 24 \text { e } 34 \text { semanas de gestação. }\end{array}$} \\
\hline \\
dose & total de doses & intervalo \\
BETAMETASONA & $12,0 \mathrm{mg} \mathrm{MM}$ & 2 & 24 horas \\
DEXAMETASONA & $6,0 \mathrm{mg} \mathrm{IM}$ & 4 & 12 horas \\
\hline
\end{tabular}

nascidos tiveram peso inferior a I 500 gramas.

Importante ressaltar que os esquemas terapêuticos preconizados não podem representar risco adicional em situações específicas onde as condições maternas ou fetais impõem intervenção imediata, face ao risco iminente de óbito ou diante de grave descompensação clínica materna. Assim sendo, éfundamental que se redobre a atenção para se registrar um possível agravamento do quadro clínico materno durante o tempo necessário do ciclo por conta das características da patologia intercorrente.

Consideramos que os resultados obtidos neste trabalho possam contribuir para a ampliação da utilização de corticóides em pacientes portadoras de síndromes hipertensivas da gestação no período pré-natal 
SASS N ET AL.

como rotina nos diversos serviços que assistem pacientes denominadas de alto risco.

Ressalte-se que a presença de hipertensão arterial representa o principal problema a ser enfrentado em termos de prematuridade eletiva em vista da insuficiência placentária instalada frequentemente nestas situações. Finalizamos com a convicção que a utilização de ciclos de corticóides neste grupo de pacientes não representa risco adicional a condição clínica materna, representando, sim, um importante diferencial em termos de sobrevida neo-natal.

\section{Agradecimentos}

Ao médico responsável pela estatística, Dr. Reinaldo José Gianini.

\section{SUMMARY}

\section{Antenatal glucocorticoid therapy AND HYPERTENSIVE PREGNANCIES: EFFECT IN MATERNAL BLOOD PRESSURE}

PURPOSE. To evaluate the behaviour of blood pressure among hypertensive pregnants during g/ucocorticoid therapy (LIGGINS scheme) in order to accelerate the maturity of fetal lung.

METHODS. In a retrospective study, 27 pregnant with a story of hypertensive disor- der, between 24 and 34 weeks, during glucocorticoid cycle were avaluated. For this matter, statistical analisis were performed using the means of systolic (SBP) and diastolic blood pressure during the therapy days, on the day before and on the day after. The results were statistically analyzed with $F$ statistic test ( significant if $p<005$ )

RESULTS. There was no statisticalvariance in blood pressure levels, neither in SBP nor in DBP. There was no difficulty in clinical controls and no change in antihypertensive drugs related to g/ucocorticoid therapy.

ConCLUSIOns. Our results show observe the safety of glucocorticoid therapy among pregnant women with hypertensive disorders related to the possible raising of the blood pressure levels. [Rev Ass Med Brasil 200।; 47(3): 255-8]

KEY wORDS: Gestational hypertension. Perinatal outcome. Glucocorticoids.

\section{REFERÊNCIAS}

I. Liggins GC, Howie RN. A controlled trial of antepartum glucocorticoid treatment for prevention of respiratory distress syndrome in premature infants. Pediatrics 1972; 50 : 5 | 5-25.

2. Crowley P, Chalmers I, Keirse MJNC. The effects of corticosteroid administration before premature delivery. Br J Obstet Gynecol 1990; 97: $11-25$.
3. NIH consensus development panel on the effect of corticosteroids for fetal maturation on perinatal outcome. Effect of corticosteroids for fetal maturation on perinatal outcomes. JAMA, 1995; 273:413-8.

4. Nochimson DJ, Petrie RH. Glucocorticoid therapy for the induction of pulmonary maturity in severely hypertensive gravid women. Am J Obstet Gynecol 1979; 133:449-53.

5. Rickie PS, Elliott JP, Freeman RK. Use of corticosteroids in pregnancy - induced hypertension. Am J Obstet Gynecol 1980; 55: 106-10.

6. Collaborative group on atenatal steroid therapy- Effect of antenatal dexamethasone therapy om prevention of respiratory distress syndrome. Am J Obstet Gynecol, 1981; | 4 |:276-87.

7. Ballard RA, Ballard PL, Creasy R, Padbury JF, Polk DH, Bracken M, et al. TRH Study Group. Respiratory disease in very-low-birthweight infants after prenatal thyrotropin - releasing hormone and glucocorticoid. Lancet 1992; 339:510-15.

8. Semchyshyn S, Zuspan PF, Cordero L. Cardiovascular response and complication of glucocorticoid therapy in hypertensive pregnancies. Am J Obstet Gynecol 1983; | 45:530-41.

9. Ruvinsky E, Douvas SG, Rhodes PG, Morrison JC. The maternal administration of dexamethasone in pregnancy induced hypertension. Am J Obstet Gynecol 1984; 149:722-6.

10. Amorin MMR. Uso de corticóide para aceleração da maturidade pulmonar fetal na pré eclampsia grave [tese]. Campinas: Faculdade de Ciências Médicas da Universidade Estadual de Campinas; 1998.

Artigo recebido: I //01/2000 Aceito para publicação: 13/03/200 I 